\title{
Hadamard Matrices with Cocyclic Core
}

\author{
Víctor Álvarez ${ }^{1, *}$, José Andrés Armario ${ }^{1}\left(\right.$, María Dolores Frau ${ }^{1} \oplus$, Félix Gudiel ${ }^{1} \oplus$, María Belén Güemes ${ }^{2} \mathbb{D}$ \\ and Amparo Osuna ${ }^{1}$ (D) \\ 1 Department of Applied Mathematics I, University of Seville, 41004 Sevilla, Spain; armario@us.es (J.A.A.); \\ mdfrau@us.es (M.D.F.); gudiel@us.es (F.G.); aosuna@us.es (A.O.) \\ 2 Department of Algebra, University of Seville, 41004 Sevilla, Spain; bguemes@us.es \\ * Correspondence: valvarez@us.es
}

check for

updates

Citation: Álvarez, V.; Armario, J.A.; Frau, M.D.; Gudiel, F.; Güemes, M.B.; Osuna, A. Hadamard Matrices with Cocyclic Core. Mathematics 2021, 9, 857. https://doi.org/10.3390/ math9080857

Academic Editor: Abdelmejid Bayad

Received: 10 February 2021

Accepted: 9 April 2021

Published: 14 April 2021

Publisher's Note: MDPI stays neutral with regard to jurisdictional claims in published maps and institutional affiliations.

\begin{abstract}
Since Horadam and de Launey introduced the cocyclic framework on combinatorial designs in the 1990s, it has revealed itself as a powerful technique for looking for (cocyclic) Hadamard matrices. Ten years later, the series of papers by Kotsireas, Koukouvinos and Seberry about Hadamard matrices with one or two circulant cores introduced a different structured approach to the Hadamard conjecture. This paper is built on both strengths, so that Hadamard matrices with cocyclic cores are introduced and studied. They are proved to strictly include usual Hadamard matrices with one and two circulant cores, and therefore provide a wiser uniform approach to a structured Hadamard conjecture.
\end{abstract}

Keywords: Hadamard matrix; circulant matrix; cocyclic matrix; difference set

MSC: 05B20; 05B10

\section{Introduction}

Hadamard matrices are square matrices of order $n$ with entries from $\{-1,1\}$ such that their rows are pairwise orthogonal. They were identified as the extremal solutions of the maximal determinant problem for square matrices with entries from the unit disc [1].

It may be straightforwardly checked that, regardless of the elemental cases $n=1$ and $n=2$, as soon as three rows have to be mutually orthogonal, this implies that $n$ is necessarily a multiple of 4 . Unexpectedly, the converse assertion (that there exists a Hadamard matrix for every order $n=4 w$ ), remains one of the most famous open centuryold problems in mathematics, the Hadamard Conjecture.

The techniques for constructing Hadamard matrices are usually organized into three types: multiplication theorems, "plug-in" methods and direct constructions [2,3]. Nevertheless, none of them has succeeded to provide a uniform method for constructing these matrices.

The cocyclic approach, as introduced in [4], tries to cast new light on this purpose. A cocyclic matrix (over a group $G$ ) consists of a matrix $(\psi(i, j))$ constructed from a binary (2-)cocycle over $G, \psi: G \times G \rightarrow\{-1,1\}$, so that:

$$
\psi(a, b) \psi(a, b \cdot c) \psi(a \cdot b, c) \psi(b, c)=1,
$$

for all $a, b, c \in G$. It is called a 2-coboundary if a map $\phi: G \rightarrow G$ exists such that $\psi(a, b)=\phi(a) \phi(b) \phi(a b)$, for every $a, b \in G$.

Accordingly, checking whether a cocyclic matrix is Hadamard translates into checking if the summation of each row (but the first) is zero [5]. This is not the only advantage of this approach. It endows the matrix with an enriched structure, which is being successfully exploited to look for Hadamard matrices [2,6,7]. Nowadays, this framework is broadening its expected horizons, since more recently even quasigroups [8] and Latin rectangles [9] have come into play. 
(Cocyclic) Hadamard matrices are intimately related to other combinatorial objects, such as designs, (almost) difference sets and (almost) perfect sequences. We next briefly recall these notions and relations, as they are introduced in $[2,10]$, since they will be relevant in the exposition of the paper. For further information, the interested reader is referred to $[2,10]$ and the references therein.

$\mathrm{A}(v, k, \lambda)$-design is a pair $\mathcal{D}=(P, B)$ consisting of a $v$-set $P=\left\{p_{1}, \ldots, p_{v}\right\}$ of points and a $v$-set $B=\left\{B_{1}, \ldots, B_{v}\right\}$ of $k$-blocks, $1<k<v$, such that each pair of distinct points is contained in exactly $\lambda$ blocks.

If $G$ is an automorphism group of $\mathcal{D}$ such that for each pair of points $p_{i}, p_{j}$ there is a unique $g \in G$ such that $g\left(p_{i}\right)=p_{j}$, and similarly for blocks, the design $\mathcal{D}$ is called regular with respect to $G$, and $G$ is called a regular group for $\mathcal{D}$.

Matricially, $\mathcal{D}$ is easily represented by its $v \times v$ incidence matrix $A_{\mathcal{D}}=\left(a_{i j}\right)$, rows and columns in correspondence with blocks and points, respectively, such that $a_{i j}=1$ if and only if $p_{j} \in B_{i}$, and 0 otherwise.

It may be straightforwardly checked that a $v \times v$ square matrix $A$ with entries 0,1 is an incidence matrix of a $(v, k, \lambda)$-design if and only if $A A^{T}=(k-\lambda) I+\lambda J$ and $A J=k J$, for $I$ the identity matrix and $J$ the all 1 s matrix.

In particular, there exists a Hadamard matrix $H$ of order $4 w$ if and only if there exists a square $(4 w-1,2 w-1, w-1)$-design $\mathcal{D}$. Actually, noting $A^{\prime}=2 A_{\mathcal{D}}-J$, the matrix obtained from $A_{\mathcal{D}}$ by replacing 0 s by -1 s, then $H=\left(\begin{array}{cc}1 & \mathbf{1} \\ \mathbf{1}^{T} & A^{\prime}\end{array}\right)$, is a Hadamard matrix, and vice versa.

Other combinatorial structures intimately related to Hadamard matrices and designs are difference sets.

A $(v, k, \lambda)$-difference set in a group $(G, \cdot)$ of order $v$ is a $k$-subset $D \subset G$ such that the $k(k-1)$-list of quotients $d_{1} d_{2}^{-1}$ of distinct elements $d_{1}, d_{2}$ of $D$ contains each non-identity element of $G$ exactly $\lambda$ times. The order of the difference set is $n=k-\lambda$. The difference set is called cyclic or abelian, if $G$ has the respective property.

Remark 1. Notice that if $D$ is $a(v, k, \lambda)$-difference set, then $G \backslash D$ defines $a(v, v-k, v-2 k+\lambda)$ difference set, the so-called complementary difference set of $D$.

Let $G$ be a group of $v$ elements, and $D \subset G$ a $k$-subset, $1<k<v$. Then $D$ is a $(v, k, \lambda)$-difference set in $G$ if and only if $\mathcal{D}=(G,\{g D: g \in G\})$ is a $(v, k, \lambda)$-design with regular group $G$, and vice versa. Therefore, as soon as a $(4 w-1,2 w-1, w-1)$-difference set exists, there exists a Hadamard matrix of order $4 w$ in turn.

Now we turn our interest to (almost) perfect sequences, as introduced in [10].

Consider a cyclic binary sequence $\left(a_{i}\right)$ with entries from $\{-1,1\}$ of period $v$, such that $a_{i+v}=a_{i}$, for $i \geq 0$. The autocorrelation of $\left(a_{i}\right)$ for shift $t$ is defined as the sum $c_{t}\left(a_{i}\right)=\sum_{i=0}^{v-1} a_{i} a_{i+t}$.

A sequence $\left(a_{i}\right)$ is called perfect when $c_{t}\left(a_{i}\right)=r$ is constant for $0<t \leq v-1$, and equals the smallest possible $\max \left|c_{t}\left(a_{i}\right)\right| \in\{0,1,2\}$, since $c_{t}(a) \equiv v \bmod 4$.

Remark 2. Notice that a perfect periodic sequence with period $v, k$ positive entries per period and autocorrelation $c_{t}\left(a_{i}\right)=r, 0<t \leq v-1$, is equivalent to a cyclic $(v, k, \lambda)$-difference set: it suffices to take $D=\left\{i: a_{i}=1,0 \leq i \leq v-1\right\}$, so that $|D \cap(D+t)|=\lambda$ and $r=v-4(k-\lambda)$.

Depending on the value $c_{t}\left(a_{i}\right)=r \in\{0, \pm 1, \pm 2\}$, the existence of perfect sequences $\left(a_{i}\right)$ is summarized in [10]. In particular:

- The case $r=0$ corresponds to circulant Hadamard matrices of order $v$, which are conjectured to exist just for $v=4$.

- The case $r=1$ corresponds to $\left(2 u^{2}+2 u+1, u^{2}, \frac{u^{2}-u}{2}\right)$-cyclic difference sets, which are conjectured to exist just for $u=1,2$. 
- There is no known example for the case $r=2$, and experimental results suggest that none might exist.

- The case $r=-2$ gives just one difference set, the $(2,1,0)$-difference set.

- The case $r=-1$ gives the so-called Paley-Hadamard difference sets, with parameters $\left(v, \frac{v-1}{2}, \frac{v-3}{4}\right)$, whose existence implies in turn the existence of Hadamard matrices of order $v+1$. These include very well known families of difference sets [10].

Remark 3. The case $r=-1$ is of particular interest in this paper, since it includes the so-called Hadamard matrices with one circulant core [11], of the form $\left(\begin{array}{ll}1 & \mathbf{1} \\ \mathbf{1} & A\end{array}\right)$, for A being a circulant matrix whose first row consists of a perfect sequence $\left(a_{i}\right)$ with $c_{t}\left(a_{i}\right)=-1,0<t \leq 4 w-2$, and $\sum_{i=0}^{4 w-2} a_{i}=-1$

When relaxing some conditions, the combinatorial structures described above may be generalized, in some sense.

For instance, given a $k$-subset $D$ of a group $(G, \cdot)$ of order $v$, it might occur that the number of occurrences of each non identity element in $G$ among the list of quotients $d_{1} d_{2}^{-1}$ of distinct elements $d_{1} \neq d_{2} \in D$ is either $\lambda$ or $\lambda+1$, not just $\lambda$. Then $D$ defines a $(v, k, \lambda, s)$-almost difference set, where $s$ denotes the amount of elements of $G \backslash\{1\}$ that appears precisely $\lambda$ times in the list $d_{1} d_{2}^{-1}$, the remaining $v-s-1$ elements of $G$ appearing exactly $\lambda+1$ times.

Almost difference sets are related to almost perfect sequences, in the following manner.

A sequence $\left(a_{i}\right)$ is called almost perfect when two possible values $r_{1}, r_{2}$ are allowed for $c_{t}\left(a_{i}\right)$, for $0<t \leq v-1$, with $\left|r_{1}\right|+\left|r_{2}\right|=4$. As noted in [10], this is equivalent to $D=\left\{i: a_{i}=1,0 \leq i \leq v-1\right\}$ being an $\left(v, k, k-\left\lfloor\frac{v}{4}\right\rfloor-1, k v-k^{2}-(k-1)\left\lfloor\frac{v}{4}\right\rfloor\right)$ almost difference set, for $k=|D|$.

As claimed in [10], computer experimentation seems to suggest that balanced (i.e., half of the entries are of opposite sign) almost perfect sequences might exist for every $v \equiv 1(\bmod 4)$. As soon as two such balanced sequences $\left(a_{i}\right)$ and $\left(b_{i}\right)$ combine to give $c_{t}\left(a_{i}\right)+c_{t}\left(b_{i}\right)=-2$, for $0<t \leq 2 w-2$, a Hadamard matrix of order $4 w$ would exist in turn: this is one of the most prolific techniques for constructing Hadamard matrices of order $4 w$, the so-called two circulant cores "plug-in" method [3,12,13].

Actually, the Hadamard matrix would consist in:

$$
\left(\begin{array}{rrrr}
-1 & -1 & \mathbf{1} & \mathbf{1} \\
-1 & 1 & \mathbf{1} & -\mathbf{1} \\
\mathbf{1} & \mathbf{1} & A & B \\
\mathbf{1} & -\mathbf{1} & B^{T} & -A^{T}
\end{array}\right)
$$

for $A$ and $B$ being the circulant matrices based on $\left(a_{i}\right)$ and $\left(b_{i}\right)$, respectively.

As a matter of fact, among the most prominent "plug-in" methods for constructing Hadamard matrices, precisely the two circulant cores technique and the Goethals-Seidel arrays [14] were the only ones that remain out of the cocyclic framework, as indicated in [15]. Actually, Goethals-Seidel arrays have been recently characterized as (pseudo)cocyclic matrices over quasigroups in [16].

Finally, we now recall the characterization of cocyclic Hadamard matrices in terms of certain difference sets and designs, as introduced in Theorem 2.4 of [17], and which is used in [6] to provide an exhaustive classification of cocyclic Hadamard equivalent matrices of orders less than 40 . For the sake of simplicity and readability, we will not detail the definition of these particular combinatorial structures, as they are not needed for the comprehension of the paper. 
Theorem 1. [17] The following statements are equivalent:

1. There is a cocyclic Hadamard matrix over $G,|G|=4 w$.

2. There is a (normal) relative ( $4 w, 2,4 w, 2 w)$-difference set in a central extension of $\langle-1\rangle$ by $G$, relative to $\langle-1\rangle$.

3. There is a divisible $(4 w, 2,4 w, 2 w)$-design, class regular with respect to $\langle-1\rangle$, and with central extension of $\langle-1\rangle$ by $G$ as a regular group of automorphisms.

The aim of this paper is to settle a theoretical background for the study of Hadamard matrices consisting of cocyclic cores, instead of circulant cores. Actually, Hadamard matrices with a cocyclic core will be shown to be placed in some sense halfway from full cocyclic Hadamard matrices and usual Hadamard matrices, as $4 w \times 4 w$ Hadamard matrices with a cocyclic core will be proven to exist if and only if there exist $(4 w-1,2 w-$ $1, w-1$ )-difference sets (Theorem 2), which are already known to imply the existence of full Hadamard matrices, as commented before.

The paper is organized as follows. In Section 2, we characterize the existence of Hadamard matrices with a $(4 w-1) \times(4 w-1)$ cocyclic core, in terms of some perfect sequences and difference sets. Section 3 is devoted to the characterization of Hadamard matrices with a $(4 w-2) \times(4 w-2)$ cocyclic core, in terms of certain almost perfect sequences and almost difference sets.

\section{Hadamard Matrices with a $(4 w-1) \times(4 w-1)$ Cocyclic Core}

As introduced in Section 1, Hadamard matrices with one circulant core consist of matrices of the type $\left(\begin{array}{ll}1 & \mathbf{1} \\ \mathbf{1} & A\end{array}\right)$, for $A$ being a circulant matrix whose first row consists of a perfect binary sequence $\left(a_{i}\right)$ with $c_{t}\left(a_{i}\right)=-1,0<t \leq 4 w-2$, and $\sum_{i=0}^{4 w-2} a_{i}=-1$. They were extensively studied in [11].

In this section we study the conditions under which a cocyclic (instead of a simply circulant) matrix $M_{\psi}$ (for $\psi$ as in (1)) may be plugged in a structure of the type:

$$
\left(\begin{array}{cc}
? & ? \\
\alpha & M_{\psi}
\end{array}\right)
$$

in order to provide a Hadamard matrix as well, for $\alpha=\left(\alpha_{g_{0}}, \ldots, \alpha_{g_{4 w-2}}\right)$, given an ordering $\left\{g_{0}=1, \ldots, g_{4 w-2}\right\}$ of $G$.

Notice that the first row of such a structure is not of interest, since every partial Hadamard matrix of size $(4 w-z) \times 4 w, 1 \leq z \leq 7$, is known to be extendable to a full $4 w \times 4 w$ Hadamard matrix [18].

Theorem 2. There exists a Hadamard matrix of order $4 w$ with a $(4 w-1) \times(4 w-1)$ cocyclic core if and only if there exists $a(4 w-1,2 w, w)$-difference set (or, equivalently, a $(4 w-1,2 w-$ $1, w-1)$-difference set).

The following sequence of lemmas will help in the task of proving this result.

Lemma 1. The first row (and column) of $M_{\psi}$ consists of a common single value, either 1 or -1 .

Proof. This is a common fact of cocyclic matrices, and it may be straightforwardly derived from Equation (1): as soon as one fixes $a=b=1 \in G$, it follows that $\psi(1,1) \cdot \psi\left(1, g_{k}\right)=1$, for every $g_{k} \in G$.

A similar argument applies to the first column, taking $b=c=1$ in (1) instead.

Remark 4. Cocylic matrices $M_{\psi}$ for which $\psi(1, j)=\psi(j, 1)=1$ are usually termed normalized. 
The following result is at the very heart of the usual cocyclic test for Hadamard matrices [2,5]. However, we will rather state it and reproduce its proof explicitly, since both of them will be extensively used in the paper.

Lemma 2. For every $g_{i}, g_{j} \in G$, the dot product of rows indexed by $g_{i}$ and $g_{j}$ in $M_{\psi}$ consists of:

$$
\sum_{g_{k} \in G} \psi\left(g_{i}, g_{k}\right) \psi\left(g_{j}, g_{k}\right)=\psi\left(g_{i} g_{j}^{-1}, g_{j}\right) \sum_{g_{k} \in G} \psi\left(g_{i} g_{j}^{-1}, g_{k}\right) .
$$

Proof. Actually, this is an alternative way to read the usual cocyclic Hadamard test of [5]. From (1), taking $a=g_{i} g_{j}^{-1}, b=j$ and $c=g_{k}$, it follows that:

$$
\psi\left(g_{i} g_{j}^{-1}, g_{j}\right) \cdot \psi\left(g_{i} g_{j}^{-1}, g_{j} g_{k}\right)=\psi\left(g_{i}, g_{k}\right) \cdot \psi\left(g_{j}, g_{k}\right) .
$$

Therefore, the dot product of rows indexed by $g_{i}$ and $g_{j}$ in $M_{\psi}$ consists of:

$$
\sum_{g_{k} \in G} \psi\left(g_{i}, g_{k}\right) \psi\left(g_{j}, g_{k}\right)=\sum_{g_{k} \in G} \psi\left(g_{i} g_{j}^{-1}, g_{j}\right) \psi\left(g_{i} g_{j}^{-1}, g_{j} g_{k}\right)=\psi\left(g_{i} g_{j}^{-1}, g_{j}\right) \sum_{g_{k} \in G} \psi\left(g_{i} g_{j}^{-1}, g_{k}\right)
$$

Lemma 3. Assume that the matrix in (3) is Hadamard. Then $\sum_{g_{k} \in G} \alpha_{g_{k}}= \pm 1$.

Proof. From Remark 4 we know that $\psi\left(g_{k}, 1\right)$ is constant (for both 1 and -1 ) for every $g_{k} \in G$. Therefore, the dot product of columns 1 and 2 of the matrix in (3) consists of:

$$
0= \pm 1 \pm \sum_{g_{k} \in G} \alpha_{g_{k}}
$$

and the result follows.

Lemma 4. Assume that the matrix in (3) is Hadamard. Then $\sum_{g_{k} \in G} \psi\left(g_{i}, g_{k}\right)=-\psi\left(g_{i}, 1\right) \cdot \alpha_{1} \cdot \alpha_{g_{i}}$, for every $g_{i} \in G \backslash\{1\}$.

Proof. Taking $g_{j}=1 \in G$ in Lemma 2, it follows that the dot product of rows indexed by $g_{i}$ and 1 in (3) consists of:

$$
0=\alpha_{g_{i}} \cdot \alpha_{1}+\psi\left(g_{i}, 1\right) \sum_{g_{k} \in G} \psi\left(g_{i}, g_{k}\right),
$$

from which the asserted result derives at once.

Lemma 5. Assume that the matrix in (3) is Hadamard. Then $\psi\left(g_{i}, g_{j}\right)=\psi\left(g_{i}, 1\right) \cdot \alpha_{1} \cdot \alpha_{g_{i}} \cdot \alpha_{g_{j}}$. $\alpha_{g_{i} g_{j}}$, for every $g_{i}, g_{j} \in G$.

Proof. From Lemma 2, the dot product of the rows indexed by $g_{i} g_{j}$ and $g_{j}$ in (3) consists of:

$$
0=\alpha_{g_{i} g_{j}} \cdot \alpha_{g_{j}}+\psi\left(g_{i}, g_{j}\right) \sum_{g_{k} \in G} \psi\left(g_{i}, g_{k}\right) .
$$

Consequently, taking into account Lemma 4,

$$
0=\alpha_{g_{i} g_{j}} \cdot \alpha_{g_{j}}-\psi\left(g_{i}, 1\right) \cdot \alpha_{1} \cdot \alpha_{g_{i}} \psi\left(g_{i}, g_{j}\right),
$$

so that $\psi\left(g_{i}, g_{j}\right)=\psi\left(g_{i}, 1\right) \cdot \alpha_{1} \cdot \alpha_{g_{i}} \cdot \alpha_{g_{j}} \cdot \alpha_{g_{i} g_{j}}$.

In the circumstances above, the following results may be straightforwardly derived. 
Corollary 1. $\psi$ is completely characterized as a 2-coboundary $\psi\left(g_{i}, g_{j}\right)=\phi\left(g_{i}\right) \phi\left(g_{j}\right) \phi\left(g_{i} g_{j}\right)$, for $\phi: G \rightarrow\{-1,1\}$ defined as $\phi\left(g_{i}\right)=\phi\left(g_{1}\right) \cdot \alpha_{1} \cdot \alpha_{g_{i}}, \phi\left(g_{1}\right)= \pm 1$.

Remark 5. Notice that it is irrelevant whether one fixes $\phi\left(g_{1}\right)$ to be either 1 or -1 . This just has to be with the (un)normalized character of the related 2-cocycle, as noted in Remark 4.

Corollary 2. $\sum_{g_{j} \in G} \alpha_{g_{j}} \alpha_{g_{i} g_{j}}=-1$, for every $g_{i} \in G$.

Proof. From Lemma 5, $\psi\left(g_{i}, g_{j}\right)=\alpha_{1} \alpha_{g_{i}} \alpha_{g_{j}} \alpha_{g_{i} g_{j}}$. Adding as $g_{j}$ runs on $G$, we obtain:

$$
-\alpha_{1} \alpha_{g_{i}}=\sum_{g_{j} \in G} \psi\left(g_{i}, g_{j}\right)=\alpha_{1} \alpha_{g_{i}} \sum_{g_{j} \in G} \alpha_{g_{j}} \alpha_{g_{i} g_{j}},
$$

from which the result derives at once.

Lemma 6. Assume that $\left(\alpha_{g_{k}}\right)$ and $M_{\psi}$ are given so that rows from 2 to $4 \mathrm{w}$ in (3) are pairwise orthogonal. Then this matrix may be completed to a full Hadamard matrix, in essentially one way (up to negation of the row).

Proof. Let $\left(s_{1}, s_{2}, \ldots, s_{4 w}\right)$ be the unknown entries of the first row of (3).

Lemma 1 guarantees that the first column in $M_{\psi}$ consists of a common constant, $\psi(1,1)$. Take $s_{2}=\psi(1,1)$, so that the second column of (3) consists of a common constant.

From the orthogonality law, the dot product of column $i \neq 2$ with the second column has to be zero. Consequently, since the second column is formed by a common constant, the summation of each column $i \neq 2$ of (3) has to be zero, as well. This characterizes uniquely the first row of the matrix (once $s_{2}$ has been fixed).

We can now prove Theorem 2.

Proof. We first prove the sufficient condition.

Assume that a Hadamard matrix of the form (3) exists, with a cocyclic core consisting of $M_{\psi}$.

In these circumstances, notice that $\left(\alpha_{g_{k}}\right)$ defines a generalized perfect sequence, in the sense that:

- It is balanced, as Lemma 3 indicates.

- A generalized autocorrelation function for $\left(\alpha_{g_{i}}\right)$ may be defined, so that shift $g_{t}$ depends on the right action of $g_{t}$ by means of the group law of $G: c_{t}\left(\alpha_{g_{k}}\right)=\sum_{g_{k} \in G} \alpha_{g_{k}} \alpha_{g_{k} g_{t}}$.

- $\quad$ Corollary 2 shows that $\left(\alpha_{g_{k}}\right)$ is perfect.

In these circumstances, the set $D=\left\{g_{k}: \alpha_{g_{k}}=1\right\}$ defines a difference set, as noted in Remark 2.

Since the sequence is balanced, it follows that $|D|=2 w$ or $|D|=2 w-1$. Furthermore, it may be straightforwardly checked that $\left|D \cap D \cdot g_{t}\right|=|D|-w$. No matter what the concrete value of $|D|$ is, a couple of complementary difference sets with parameters (4w$1,2 w, w)$ and $(4 w-1,2 w-1, w-1)$ are thus defined (see Remark 1 ).

Conversely, starting from such a difference set, as noted in Remark 2, the process works the other way around, and the proof ends, as soon as the matrix so obtained may be extended to a full Hadamard matrix, attending to Lemma 6.

Example 1. Consider the multiplicative cyclic group of order $3, G=\left(C_{3}, \cdot\right)=\left\langle a: a^{3}=1\right\rangle$, endowed with the natural ordering $\left\{1, a, a^{2}\right\}$.

The subset $D=\left\{a, a^{2}\right\}$ defines $a(3,2,1)$-difference set in $G$, since $a \cdot a^{-2}=a^{2}$ and $a^{2} \cdot a^{-1}=a$.

Let us consider the sequence $\alpha=\left(\alpha_{g}\right)_{g \in D}=(-1,1,1)$, such that $\alpha_{g}=1$ if $g \in D$, and $\alpha_{g}=-1$ otherwise. 
By construction, $\left(\alpha_{g}\right)$ defines a perfect sequence. Actually:

- $\quad \alpha_{1} \cdot \alpha_{1 \cdot a}+\alpha_{a} \cdot \alpha_{a \cdot a}+\alpha_{a^{2}} \cdot \alpha_{a^{2} \cdot a}=-1$.

- $\quad \alpha_{1} \cdot \alpha_{1 \cdot a^{2}}+\alpha_{a} \cdot \alpha_{a \cdot a^{2}}+\alpha_{a^{2}} \cdot \alpha_{a^{2} \cdot a^{2}}=-1$.

Consider the map $\phi:\left(C_{3}, \cdot\right) \rightarrow\{-1,1\}$ defined as $\phi(1)=1, \phi(a)=\alpha_{1} \cdot \alpha_{a}=-1$, $\phi\left(a^{2}\right)=\alpha_{1} \cdot \alpha_{a^{2}}=-1$. Let $\psi: C_{3} \times C_{3} \rightarrow\{-1,1\}$ be the 2-coboundary defined as $\psi(i, j)=$ $\phi(i) \phi(j) \phi(i \cdot j)$, so that $M_{\psi}$ reads as:

$$
\left(\begin{array}{rrr}
1 & 1 & 1 \\
1 & -1 & 1 \\
1 & 1 & -1
\end{array}\right) .
$$

Plugging this matrix in (3), we get:

$$
\left(\begin{array}{r|rrr}
? & ? & ? & ? \\
\hline-1 & 1 & 1 & 1 \\
1 & 1 & -1 & 1 \\
1 & 1 & 1 & -1
\end{array}\right) .
$$

This matrix may be completed to form a full Hadamard matrix, as soon as one takes its first row to be $(-1,1,-1,-1)$, attending to Lemma 6 .

Remark 6. Notice that if one negates both of the third and fourth rows and columns of the Hadamard matrix of Example 1, one obtains the Hadamard equivalent matrix:

$$
\left(\begin{array}{r|rrr}
-1 & 1 & 1 & 1 \\
\hline-1 & 1 & -1 & -1 \\
-1 & -1 & -1 & 1 \\
-1 & -1 & 1 & -1
\end{array}\right),
$$

which is indeed a Hadamard matrix with a back-circulant core. This may be transformed into a Hadamard equivalent matrix consisting of a usual circulant core, as soon as rows are permuted accordingly. This is not a casual fact, as the following result asserts.

Proposition 1. Any Hadamard matrix with a circulant core is Hadamard equivalent to a Hadamard matrix with a cocyclic core.

Proof. As noted in Remark 3 (see [11] for details), any $4 w \times 4 w$ Hadamard matrix $H$ with a circulant core $H_{C}$ is in one-to-one correspondence with the cyclic $(4 w, 2 w, w)$-difference set $D=\left\{a^{i}: H_{C}(1, i-1)=1\right\} \subset\left(C_{4 w-1}, \cdot\right)$, consisting of the elements that correspond to the positions at which the $1 \mathrm{~s}$ of the first row of $H_{C}$ are located.

As illustrated in Example 1, starting from such a cyclic difference set, application of the constructive proof of Theorem 2 provides a Hadamard matrix with a cocyclic core $M_{\psi}$. Furthermore, Corollary 1 shows that the map $\phi$ underlying $M_{\psi}$, as defined, provides a cocyclic core, which is Hadamard equivalent to the initial circulant core. To check this, it suffices to negate both the sets of rows and columns indexed by the elements in $D$, and reorder the rows from bottom to top.

A question arises in a natural way: is there any advantage in looking for Hadamard matrices with cocyclic cores instead of Hadamard matrices with circulant cores?

Proposition 2. Hadamard matrices with cocyclic cores strictly include Hadamard matrices with one circulant core.

Proof. Hadamard matrices of order $4 w$ with a circulant core are in one-to-one correspondence with cyclic $(4 w-1,2 w-1, w-1)$-difference sets.

However, Hadamard matrices or order $4 w$ with a cocyclic core are in one-to-one correspondence with $(4 w-1,2 w-1, w-1)$-difference sets, which are not necessarily cyclic. 
Actually, there are orders $4 w$ for which no such cyclic difference sets exist, but as yet, general difference sets do exist.

Example 2. Consider the case $4 w=28$, for instance.

On the one hand, Theorem 2 in [11] states that there are no $28 \times 28$ Hadamard matrices with a circulant core. Consequently, no cyclic $(27,13,6)$-different set can exist.

On the other hand, $(27,13,6)$-difference sets do exist: as noted in [19],

$$
D=\left\{1, a, a^{2}, b, a b, b^{2}, c, a c, b c, a c^{2}, a^{2} b c^{2}, b^{2} c^{2}, a^{2} b^{2} c^{2}\right\}
$$

constitutes $a(27,13,6)$-difference set in $G=\left(C_{3} \times C_{3} \times C_{3}, \cdot\right)=\left\langle a, b, c: a^{3}=b^{3}=c^{3}=1\right\rangle$, with ordering $a^{i_{1}} b^{j_{1}} c^{k_{1}}<a^{i_{2}} b^{j_{2}} c^{k_{2}}$ if and only if $k_{1}<k_{2}$, or $k_{1}=k_{2}$ and $j_{1}<j_{2}$, or $\left(j_{1}, k_{1}\right)=$ $\left(j_{2}, k_{2}\right)$ and $i_{1}<i_{2}$.

Let $u$ consider the sequence $\alpha=\left(\alpha_{g}\right)_{g \in D}$, such that $\alpha_{g}=1$ if $g \in D$, and $\alpha_{g}=-1$ otherwise.

Since $\alpha_{1}=1$, consider the map $\phi: G \rightarrow\{-1,1\}$ defined as $\phi(g)=\alpha_{g}$, for $g \in G$. Let $\psi: G \times G \rightarrow\{-1,1\}$ be the 2-coboundary defined as $\psi(i, j)=\phi(i) \phi(j) \phi(i \cdot j)$. Plugging $\left(\alpha_{g}\right)$ and $M_{\psi}$ in (3) and completing with regards to Lemma 6 leads to a $28 \times 28$ Hadamard matrix with a cocyclic core.

\section{Hadamard Matrices with a $(4 w-2) \times(4 w-2)$ Cocyclic Core}

Hadamard matrices with two circulant cores $[3,12,13]$ come from a new "plug-in" technique, which extends the underlying idea applied when constructing Hadamard matrices with one circulant core. In this occasion, properly fixing two rows and columns, a full Hadamard matrix may be constructed as soon as two $(2 w-1) \times(2 w-1)$ circulant matrices $A$ and $B$ exist, satisfying the constraint:

$$
A A^{T}+B B^{T}=4 w I_{2 w-1}-2 J_{2 w-1},
$$

in which case the matrix:

$$
\left(\begin{array}{rrrr}
-1 & -1 & \mathbf{1} & \mathbf{1} \\
-1 & 1 & \mathbf{1} & -\mathbf{1} \\
\mathbf{1} & \mathbf{1} & A & B \\
\mathbf{1} & -\mathbf{1} & B^{T} & -A^{T}
\end{array}\right)
$$

would be Hadamard.

As commented in Section 1 , this is the case if and only if the first rows of $A$ and $B$, $\left(a_{i}\right)$ and $\left(b_{i}\right)$, define two balanced almost perfect sequences with $c_{t}\left(a_{i}\right)+c_{t}\left(b_{i}\right)=-2$, for $0<t \leq w-1$.

In this section we study the conditions under which a cocyclic matrix $M_{\psi}$ (for $\psi$ as in (1)) may be plugged in a structure of the type:

$$
\left(\begin{array}{ccc}
? & ? & ? \\
\alpha & \beta & M_{\psi}
\end{array}\right)
$$

in order to provide a Hadamard matrix as well, for $\alpha=\left(\alpha_{g_{0}}, \ldots, \alpha_{g_{4 w-3}}\right)$ and $\beta=\left(\beta_{g_{0}}, \ldots, \beta_{g_{4 w-3}}\right)$, given an ordering $\left\{g_{0}=1, \ldots, g_{4 w-3}\right\}$ of $G$.

As noted in Section 1, as soon as the rows from 3 to $4 w$ are pairwise orthogonal, the matrix can be completed to a full Hadamard matrix [18]. Therefore we will focus on the rows from 3 to $4 w$ in (5).

In what follows, the notation used in Section 2 still applies, so that the rows from 3 to $4 w$ in (5) will be indexed by the corresponding elements in G. In particular, it is remarkable that the row indexed by $1 \in G$ corresponds to the third row in (5).

Furthermore, attending to Hadamard equivalence, up to negation of columns 1 or 2 in (5), without loss of generality we may assume that $\alpha_{1}=\beta_{1}=\psi(1,1)$, so that the third row in (5) (that is indexed by $1 \in G$ ) consists of the constant vector $\psi(1,1) \cdot \mathbf{1}$. 
We now determine a collection of necessary and sufficient conditions for (5) being a Hadamard matrix with a cocyclic core.

Lemma 7. Assume that (5) is a Hadamard matrix with a cocyclic core $M_{\psi}$. Then $\sum_{g \in G} \psi(h, g) \in$ $\{-2,0,2\}$, for all $h \in G \backslash\{1\}$. Moreover,

- $\sum_{g \in G} \psi(h, g)=0$ if and only if $\left(\alpha_{h}, \beta_{h}\right)=(-1,1) \cdot s_{h}$, for some (undetermined by now) $s_{h} \in\{1,-1\}$.

- $\quad 0 \neq \sum_{g \in G} \psi(h, g)= \pm 2$ if and only if $\left(\alpha_{h}, \beta_{h}\right)=(-1,-1) \cdot s_{h}$, for $s_{h}=\frac{1}{2} \sum_{g \in G} \psi(h, g)$.

Proof. Let $h \in G \backslash\{1\}$.

Taking into account the orthogonality constraint, it follows that the dot product 〈row $h$, row 1 ) of rows indexed by $h$ and $1 \in G$ in (5) must be zero. Therefore, from Lemma 2,

$$
0=\alpha_{h} \alpha_{1}+\beta_{h} \beta_{1}+\psi(h, 1) \cdot \sum_{g \in G} \psi(h, g) .
$$

Since $\alpha_{1}=\beta_{1}=\psi(1,1)=\psi(h, 1)$, we get $\sum_{g \in G} \psi(h, g)=-\alpha_{h}-\beta_{h}$ and the result follows.

Corollary 3. Assume that (5) is a Hadamard matrix with a cocyclic core $M_{\psi}$, such that $\sum_{g \in G} \psi(h, g)$ $\in\{-2,2\}$, for all $h \in G \backslash\{1\}$. Then $\psi$ is a 2-coboundary and $w=1$.

Proof. For every $g, h \in G$, attending to Lemma 2, the dot product of rows indexed by $g h$ and $h$ is:

$$
0=2 s_{g h} s_{h}+\psi(g, h) s_{g},
$$

so that $\psi(g, h)=-s_{g} s_{h} s_{g h}$ is actually a 2-coboundary $(\psi(g, h)=\phi(g) \phi(h) \phi(g h)$, for $\phi: G \rightarrow\{1,-1\}$ defined as $\left.\phi(g)=-s_{g}\right)$.

Furthermore, the summation of the row indexed by $g$ in $M_{\psi}$ is $2 s_{g}=\sum_{h \in G} \psi(g, h)=$ $-s_{g} \sum_{h \in G} s_{h} s_{g h}$, so that $\sum_{h \in G} s_{h} s_{g h}=-2$ and $\left(s_{g}\right)_{g \in G}$ defines a perfect sequence. It is known (see [10] for instance) that the only difference set corresponding to a perfect sequence of this type is the trivial $(2,1,0)$-difference set, and hence $w=1$.

Remark 7. Consequently, from now on, we assume that there are elements $h \in G$ for which $\sum_{g \in G} \psi(h, g)=0$. Actually, in these circumstances, notice that $\psi$ becomes a quasi-orthogonal cocycle, as introduced in [20].

Lemma 8. In the circumstances above, the elements in $G \backslash\{1\}$ may be organized into two subsets, $S_{0}=\left\{h \in G \backslash\{1\}: \sum_{g \in G} \psi(h, g)=0\right.$, and $\bar{S}_{0}=(G \backslash\{1\}) \backslash S_{0}$, such that:

1. $h \in S_{0} \Longleftrightarrow h^{-1} \in S_{0}$. Accordingly, $h \in \bar{S}_{0} \Longleftrightarrow h^{-1} \in \bar{S}_{0}$.

2. For all $g \in S_{0}, g \cdot S_{0} \subseteq G \backslash S_{0}$. Therefore, $\left|S_{0}\right| \leq\left|G \backslash S_{0}\right|$.

3. For all $g \in G \backslash S_{0}, g \cdot\left(G \backslash S_{0}\right) \subseteq G \backslash S_{0}$. Therefore, $\left|G \backslash S_{0}\right| \leq\left|S_{0}\right|$.

4. For all $g \in S_{0}, g \cdot\left(G \backslash S_{0}\right) \subseteq S_{0}$ and $\left(G \backslash S_{0}\right) \cdot g \subseteq S_{0}$. 
Proof. We proceed with the demonstration point by point.

1. For every $h \in G \backslash\{1\}$, since $\langle$ row 1 , row $h\rangle=\langle$ row $h$, row 1$\rangle$. Therefore $\psi\left(h^{-1}, h\right) \sum_{g \in G} \psi\left(h^{-1}, g\right)=\psi(h, 1) \sum_{g \in G} \psi(h, g)$, and the result follows.

2. Let $g, h \in S_{0}$. Then $h^{-1} \in S_{0}$ as well. Now:

$$
0=\left\langle\text { row } g, \text { row } h^{-1}\right\rangle=\alpha_{g} \alpha_{h^{-1}}+\alpha_{g} \alpha_{h^{-1}}+\psi(g h, h) \cdot \sum_{k \in G} \psi(g h, k) .
$$

Therefore $g h \in G \backslash S_{0}$.

3. Let $g, h \in G \backslash S_{0}$. Then $h^{-1} \in G \backslash S_{0}$ as well. Now:

$$
0=\left\langle\text { row } g, \text { row } h^{-1}\right\rangle=\alpha_{g} \alpha_{h^{-1}}+\alpha_{g} \alpha_{h^{-1}}+\psi(g h, h) \cdot \sum_{k \in G} \psi(g h, k) .
$$

Therefore $g h \in G \backslash S_{0}$.

4. Let $g \in S_{0}$ and $h \in G \backslash S_{0}$. Then $h^{-1} \in G \backslash S_{0}$ as well. Now:

$$
0=\left\langle\text { row } g, \text { row } h^{-1}\right\rangle=\alpha_{g} \alpha_{h^{-1}}-\alpha_{g} \alpha_{h^{-1}}+\psi(g h, h) \cdot \sum_{k \in G} \psi(g h, k) .
$$

Therefore, $g h \in S_{0}$.

Corollary 4. In the circumstances above, $\left|S_{0}\right|=\left|G \backslash S_{0}\right|=2 w-1, G \backslash S_{0}$ is a normal subgroup of $G$ and $G /\left(G \backslash S_{0}\right) \simeq C_{2}$.

Proof. This is a straightforward consequence of Lemma 8.

Remark 8. In the circumstances above, one may define a new ordering for the elements in $G$, such that the last $2 w-1$ elements correspond to $S_{0}$. For instance, when any $g \in S_{0}$ are fixed, such an ordering would be $\left\{1=n_{1}, \ldots, n_{2 w-1}, g, g n_{2}, \ldots, g n_{2 w-1}\right\}$.

Corollary 5. In the circumstances above, for $h \in \bar{S}_{0}$ and $g \in G, \psi(h, g)=-s_{h} s_{g} s_{h g}$. Furthermore, $\sum_{g \in G} s_{g} s_{h g}=-2$.

Proof. Let $h \in \bar{S}_{0}$ and $g \in G$. Consider the dot product of rows indexed by $h g$ and $g$ in (5). From Lemma 8, depending on whether:

- $g \in G \backslash S_{0}$, then $h g \in G \backslash S_{0}$. Attending to Lemma 7, then:

$$
0=\langle\text { row } h g, \text { row } g\rangle=2 s_{h g} s_{g}+\psi(h, g) \sum_{k \in G} \psi(h, k)=2 s_{h g} s_{g}+2 s_{h} \psi(h, g) .
$$

- $g \in S_{0}$, then $h g \in S_{0}$. Attending to Lemma 7, then:

$$
0=\langle\text { row } h g, \text { row } g\rangle=2 s_{h g} s_{g}+\psi(h, g) \sum_{k \in G} \psi(h, k)=2 s_{h g} s_{g}+2 s_{h} \psi(h, g) .
$$

Whichever is the case, for $h \in \bar{S}_{0}$ and $g \in G$, it is satisfied that $\psi(h, g)=-s_{h} s_{g} s_{h g}$. Taking a summation as $g$ runs in $G$, we get $2 s_{h}=\sum_{g \in G} \psi(h, g)=-s_{h} \sum_{g \in G} s_{g} s_{h g}$. The result follows immediately.

We next summarize these results.

Theorem 3. Let (5) be a matrix with a cocyclic core $M_{\psi}$ over $G$. Then it is Hadamard if and only if the following conditions are satisfied: 
- $\quad G$ contains a normal subgroup $N$ such that $G / N \simeq C_{2}$.

- There is a binary sequence $\left(s_{g}\right)_{g \in G}$ such that:

- For every $h \in N, \psi(h, g)=-s_{h} s_{g} s_{h g}$ and $\sum_{g \in G} s_{g} s_{h g}=-2$.

- For every $k \in G \backslash N, \psi(k, g)=-s_{k} s_{g} s_{k g} \varphi(k, g)$ (for some 2-cocycle $\varphi: G \times G \rightarrow$ $\{1,-1\}$ such that $\varphi(h, g)=1$ for all $h \in N)$, and $\sum_{g \in G} s_{g} s_{k g} \varphi(g, k)=0$.

Even though it might seem difficult to meet the hypothesis described in Theorem 3 altogether, we next describe a family of Hadamard matrices with a cocyclic core.

Proposition 3. Hadamard matrices with two circulant cores are Hadamard equivalent to Hadamard matrices with a cocyclic core over $D_{4 w-2}$.

Proof. Consider the group $D_{4 w-2}=\left\langle a, b: a^{2 w-1}=b^{2}=(a b)^{2}=1\right\rangle$, with ordering $G=\left\{1, a, \ldots, a^{2 w-2}, b, \ldots, a^{2 w-2} b\right\}$. Notice that $N=\langle a\rangle$ is a normal subgroup of $G$, and that every element $k \in G \backslash N$ is of order 2, that is $k^{2}=1$. Consider the 2-cocycle $\varphi: G \times G \rightarrow\{1,-1\}$ defined as $\varphi(h, g)=-1$ if and only if both $h, g \in G \backslash N$, and 1 otherwise.

Attending to the description of cocyclic matrices over $D_{4 w-2}$ developed in $[21,22]$, it may be straightforwardly derived that the two-circulant core structure of (2) is Hadamard equivalent to a cocyclic matrix over $D_{4 w-2}$.

More concretely, consider two balanced sequences $\left(a_{i}\right)$ and $\left(b_{i}\right)$ such that $\sum a_{i} \cdot a_{i+t}+$ $\sum b_{i} \cdot b_{i+t}=-2$, for $0<t \leq w-1$. Take $s_{a^{i}}=-a_{i}$ and $s_{a^{i} b}=-b_{i}$, for $0 \leq i \leq 2 w-2$.

Now, consider the subset $C \subset G$ of indices at which the positive entries of the sequence $\left(s_{i}\right)$ (i.e., negative entries of $\left.\left(\left(a_{i}\right) \mid\left(b_{i}\right)\right)\right)$ occur, and select the corresponding subset of elementary coboundaries $\partial_{c}$, so that $\psi=\varphi \cdot \prod_{c \in C} \partial_{c}$. For the sake of convenience, it might be appropriate to recall that these elementary coboundaries are commonly defined as $\partial_{g}(h, k)=\delta_{g}(h) \delta_{g}(k) \delta_{g}(h k)$, for $\delta_{i}(j)=-1$ if and only if $i=j$, and 1 otherwise.

Actually, as indicated in [21,22], the process of successively negating the row and column indexed by $c \in C$ in $M_{\psi}$, ends in a Hadamard equivalent matrix with two back circulant cores similar to (2). Permuting the rows accordingly, the proper core of the matrix (2) may be obtained.

Now, consider the conditions $\sum a_{i} \cdot a_{i+t}+\sum b_{i} \cdot b_{i+t}=-2$, for $0<t \leq w-1$, which guarantee the orthogonal constraint of $(2)$ in $[12,13]$. These are straightforwardly translated to the conditions $\sum_{g \in G} s_{g} s_{h g}=-2$, for every $h \in N$, of Theorem 3 .

Notice that, as defined, the constraints $\sum_{g \in G} s_{g} s_{k g} \varphi(g, k)=0$, for every $k \in G \backslash N$, are naturally satisfied. Actually,

$$
\sum_{g \in G} s_{g} s_{k g} \varphi(g, k)=\sum_{h \in N} s_{h} s_{k h}-\sum_{g \in G \backslash N} s_{g} s_{k g}=0,
$$

since there is a one-to-one correspondence from $N$ to $k N=G \backslash N$, taking $h \in N$ on $g_{h}=k h$; so that each term $s_{h} s_{k h}$ on the left-hand side of the equation cancels with the corresponding term on the right-hand side, as $-s_{g_{h}} s_{k g_{h}}=-s_{k h} s_{k^{2} h}=-s_{k h} s_{h}$.

Example 3. For instance, consider the $8 \times 8$ Hadamard matrix with two circulant cores of the form (2), which arises from the sequences $\left(a_{i}\right)=\left(b_{i}\right)=(1,1,-1)$, with $a_{0} a_{1}+a_{1} a_{2}+a_{2} a_{0}=-1$ : 


$$
H=\left(\begin{array}{rr|rrr|rrr}
-1 & -1 & 1 & 1 & 1 & 1 & 1 & 1 \\
-1 & 1 & 1 & 1 & 1 & -1 & -1 & -1 \\
\hline 1 & 1 & 1 & 1 & -1 & 1 & 1 & -1 \\
1 & 1 & -1 & 1 & 1 & -1 & 1 & 1 \\
1 & 1 & 1 & -1 & 1 & 1 & -1 & 1 \\
\hline 1 & -1 & 1 & -1 & 1 & -1 & 1 & -1 \\
1 & -1 & 1 & 1 & -1 & -1 & -1 & 1 \\
1 & -1 & -1 & 1 & 1 & 1 & -1 & -1
\end{array}\right)
$$

Consider the dihedral group $\left.D_{6}=\angle a, b: a^{3}=b^{2}=(a b)^{2}=1\right\rangle$, with ordering $\left\{1, a, a^{2}, b, a b, a^{2} b\right\}$, normal subgroup $N=\left\{1, a, a^{2}\right\}$ and the subset $S_{0}=\left\{b, a b, a^{2} b\right\}$. Let $C=\left\{a^{2}, a^{2} b\right\}$ and consider the 2-cocycle $\psi: D_{6} \times D_{6} \rightarrow\{1,-1\}$ given by $\psi=\phi \varphi$, for $\phi=\partial_{a^{2}} \partial_{a^{2} b}$ and $\varphi(g, h)=-1$ if and only if both $g, h \in S_{0}$. The related cocyclic matrix is then:

$$
M_{\psi}=\left(\begin{array}{rrrrrr}
1 & 1 & 1 & 1 & 1 & 1 \\
1 & -1 & -1 & 1 & -1 & -1 \\
1 & -1 & 1 & 1 & -1 & 1 \\
1 & -1 & -1 & -1 & 1 & 1 \\
1 & 1 & 1 & -1 & -1 & -1 \\
1 & -1 & 1 & -1 & 1 & -1
\end{array}\right) .
$$

Since $\phi=\partial_{a^{2}} \partial_{a^{2} b}$, we define $s_{1}=-1, s_{a}=-1, s_{a^{2}}=1, s_{b}=-1, s_{a b}=-1, s_{a^{2} b}=1$, which is consistent with Lemma 7 and Proposition 3.

We may therefore extend $M_{\psi}$ to a full Hadamard matrix of the form (5) with a cocyclic core $M_{\psi}$, as soon as one takes $\alpha=\left(-s_{1},-s_{a},-s_{a^{2}},-s_{b},-s_{a b},-s_{a^{2} b}\right)=(1,1,-1,1,1,-1)$ and $\beta=\left(-s_{1},-s_{a},-s_{a^{2}}, s_{b}, s_{a b}, s_{a^{2} b}\right)=(1,1,-1,-1,-1,1)$ :

$$
H_{\psi}=\left(\begin{array}{rr|rrr|rrr}
1 & -1 & 1 & 1 & -1 & 1 & 1 & -1 \\
-1 & 1 & 1 & 1 & -1 & -1 & -1 & 1 \\
\hline 1 & 1 & 1 & 1 & 1 & 1 & 1 & 1 \\
1 & 1 & 1 & -1 & -1 & 1 & -1 & -1 \\
-1 & -1 & 1 & -1 & 1 & 1 & -1 & 1 \\
\hline 1 & -1 & 1 & -1 & -1 & -1 & 1 & 1 \\
1 & -1 & 1 & 1 & 1 & -1 & -1 & -1 \\
-1 & 1 & 1 & -1 & 1 & -1 & 1 & -1
\end{array}\right) .
$$

Notice that starting from $H_{\psi}$ and negating both rows and columns 5 (indexed by $a^{2}$ ) and 8 (indexed by $a^{2} b$ ), and then permuting rows $(4,5)$ to be ordered as $(5,4)$, ends with the matrix $H$ above, as claimed in Proposition 3.

Nevertheless, Hadamard matrices with a cocyclic core strictly include those Hadamard matrices with a circulant core. For the sake of completeness, we conclude the paper by describing an example of a Hadamard matrix with a cocyclic core over a group not isomorphic to any dihedral group, which supports this claim.

Example 4. Consider the group $G=C_{3} \times C_{2}=\left\langle a, b: a^{3}=b^{2}=1\right\rangle$. The subgroup $N=\langle a\rangle$ is normal in $G$.

Take $\left(s_{i}\right)_{i \in G}=(-1,-1,1,-1,-1,1)$. It may be readily checked that:

- $\quad \sum_{g \in G} s_{g} s_{a \cdot g}=s_{1} s_{a}+s_{a} s_{a^{2}}+s_{a^{2}} s_{1}+s_{b} s_{a b}+s_{a b} s_{a^{2} b}+s_{a^{2} b} s_{b}=-2$.

- $\quad \sum_{g \in G} s_{g} s_{a^{2} \cdot g}=s_{1} s_{a^{2}}+s_{a} s_{1}+s_{a^{2}} s_{a}+s_{b} s_{a^{2} b}+s_{a b} s_{b}+s_{a^{2} b} s_{a b}=-2$.

- $\quad \sum_{g \in G} s_{g} s_{b \cdot g}=s_{1} s_{b}+s_{a} s_{a b}+s_{a^{2}} s_{a^{2} b}-s_{b} s_{1}-s_{a b} s_{a}-s_{a^{2} b^{2}} s_{a^{2}}=0$.

- $\sum_{g \in G} s_{g} s_{a b \cdot g}=s_{1} s_{a b}+s_{a} s_{a^{2} b}+s_{a^{2}} s_{b}-s_{b} s_{a}-s_{a b} s_{a^{2}}-s_{a^{2} b} s_{1}=0$. 
- $\quad \sum_{g \in G} s_{g} s_{a^{2} b \cdot g}=s_{1} s_{a^{2} b}+s_{a} s_{b}+s_{a^{2}} s_{a b}-s_{b} s_{a^{2}}-s_{a b} s_{1}-s_{a^{2} b} s_{a}=0$.

Accordingly, consider the 2-cocycle $\psi=\varphi \cdot \partial_{a^{2}} \partial_{a^{2} b}$, for $\varphi(g, h)=-1$ if and only if both $g, h \in G \backslash N$. By construction, the matrix $M_{\psi}$ may be extended to a full Hadamard matrix with a cocyclic core,

$$
\left(\begin{array}{rr|rrrrrr}
1 & -1 & 1 & 1 & -1 & -1 & -1 & 1 \\
-1 & -1 & 1 & 1 & -1 & 1 & 1 & -1 \\
\hline 1 & 1 & 1 & 1 & 1 & 1 & 1 & 1 \\
1 & 1 & 1 & -1 & -1 & 1 & -1 & -1 \\
-1 & -1 & 1 & -1 & 1 & 1 & -1 & 1 \\
-1 & 1 & 1 & 1 & 1 & -1 & -1 & -1 \\
-1 & 1 & 1 & -1 & -1 & -1 & 1 & 1 \\
1 & -1 & 1 & -1 & 1 & -1 & 1 & -1
\end{array}\right) .
$$

\section{Conclusions and Further Work}

In this paper we have explored the notion of Hadamard matrices with a cocyclic core, inspired by a series of papers [3,11-13] on Hadamard matrices with circulant cores.

Actually, we have described two new "plug-in" techniques for constructing Hadamard matrices, which strictly include those based on circulant cores. They depend on the existence of certain (almost) perfect sequences and (almost) difference sets, which will be our concern in the future.

Author Contributions: Conceptualization, software and writing—original draft preparation, V.Á.; methodology, formal analysis and writing-review and editing, J.A.A. and F.G.; validation, resources, data curation, visualization, supervision and project administration, M.D.F., M.B.G. and A.O.; investigation, V.Á. and J.A.A.; funding acquisition, J.A.A. All authors have read and agreed to the published version of the manuscript.

Funding: This work is partially supported by the Research Project FQM-016 from Junta de Andalucía.

Institutional Review Board Statement: Not applicable.

Informed Consent Statement: Not applicable.

Data Availability Statement: Not applicable.

Acknowledgments: We would like to thank the anonymous reviewers for their suggestions and comments, which have contributed to an improvement of the paper. We also would like to thank the Guest Editor of this special volume for giving us the opportunity of contributing with this paper.

Conflicts of Interest: The authors declare no conflict of interest.

\section{References}

1. Hadamard, J. Résolution d'une question relative aux determinants. Bull. Sci. Math. 1893, 17, 240-246.

2. Horadam, K.J. Hadamard Matrices and Their Applications; Princeton University Press: Princeton, NJ, USA, 2007.

3. Kotsireas, I.S. Structured Hadamard Conjecture, Number Theory and Related Fields. In Springer Proceedings in Mathematics and Statistics; Borwein, J., Shparlinski, I., Zudilin, W., Eds.; Springer: Berlin/Heidelberg, Germany, 2013; Volume 43, pp. $215-217$.

4. Horadam, K.J.; de Launey, W. Cocyclic development of designs. J. Algebraic Combin. 1993, 2, 267-290; Erratum. J. Algebraic Combin. 1994, 3, 129. [CrossRef]

5. Horadam, K.J.; de Launey, W. Generation of cocyclic Hadamard matrices. Math. Appl. 1995, 325, $279-290$.

6. Ó Catháin, P.; Röder, M. The cocyclic Hadamard matrices of order less than 40. Des. Codes Cryptogr. 2011, 58, 73-88. [CrossRef]

7. De Launey, W.; Flannery, D. Algebraic Design Theory; American Mathematical Society: Providence, RI, USA, 2011.

8. Álvarez, V.; Falcón, R.M.; Frau, M.D.; Gudiel, F.; Güemes, M.B. Cocyclic Hadamard matrices over Latin rectangles. Eur. J. Comb. 2019, 79, 74-96. [CrossRef]

9. Falcón, R.M.; Álvarez, V.; Frau, M.D.; Gudiel, F.; Güemes, M.B. Pseudococyclic partial Hadamard matrices over Latin rectangles. Mathematics 2021, 9, 113. [CrossRef]

10. Arasu, K.T. Sequences and arrays with desirable correlation properties. In Information Security, Coding Theory and Related Combinatorics; Crnković, D., Tonchev, V., Eds.; IOS Press: Amsterdam, The Netherlands, 2011; pp. 136-171. 
11. Kotsireas, I.S.; Koukouvinos, C.; Seberry, J. Hadamard ideals and Hadamard matrices with circulant core. J. Comb. Math. Comb. Comput. 2006, 57, 47-63. [CrossRef]

12. Fletcher, R.J.; Gysin, M.; Seberry, J. Application of the discrete Fourier transform to the search for generalised Legendre pairs and Hadamard matrices. Australas. J. Combin. 2001, 23, 75-86.

13. Kotsireas, I.S.; Koukouvinos, C.; Seberry, J. Hadamard ideals and Hadamard matrices with two circulant cores. Eur. J. Combin. 2006, 27, 658-668. [CrossRef]

14. Goethals, J.M.; Seidel, J.J. Orthogonal matrices with zero diagonal. Can. J. Math. 1967, 19, 1001-1010. [CrossRef]

15. Ó Catháin, P. Group Actions on Hadamard Matrices. Master's Thesis, National University of Ireland, Galway, Ireland, 2008.

16. Álvarez, V.; Armario, J.A., Falcón, R.M.; Frau, M.D.; Gudiel, F.; Güemes, M.B.; Osuna, A. On cocyclic Hadamard matrices over Goethals-Seidel loops. Mathematics 2020, 8, 24. [CrossRef]

17. De Launey, W.; Flannery, D.L.; Horadam, K.J. Cocyclic Hadamard matrices and difference sets. Discrete Appl. Math. 2000, 102, 47-61. [CrossRef]

18. Verheiden, E. Integral and rational completions of combinatorial matrices. J. Combin. Theory Ser. A 1978, 25, 267-276. [CrossRef]

19. Kibler, R.E. A summary of noncyclic difference sets, $k<20$. J. Combin. Theory Ser. A 1978, 25, 62-67.

20. Armario, J.A.; Flannery, D. On quasi-orthogonal cocycles. J. Comb. Des. 2018, 26, 401-411. [CrossRef]

21. Ãflvarez, V.; Armario, J.A.; Frau, M.D.; Real, P. A system of equations for describing cocyclic Hadamard matrices. J. Comb. Des. 2008, 16, 276-290. [CrossRef]

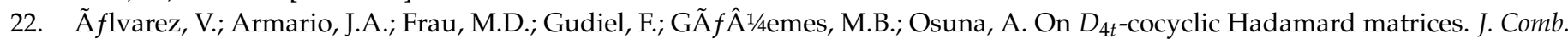
Des. 2016, 24, 352-368. [CrossRef] 